\title{
Protostellar clusters in intermediate mass (IM) star forming regions
}

\author{
A. Fuente ${ }^{1}$, C. Ceccarelli ${ }^{2}$, R. Neri ${ }^{3}$, T. Alonso-Albi ${ }^{1}$, P. Caselli ${ }^{4,5}$, \\ D. Johnstone ${ }^{6,7}$, E. F. van Dishoeck ${ }^{8}$, and F. Wyrowski ${ }^{9}$
}

\author{
1 Observatorio Astronómico Nacional (OAN), Apdo. 112, 28803 Alcalá de Henares (Madrid), Spain \\ e-mail: a. fuente@oan.es \\ ${ }^{2}$ Laboratoire d'Astrophysique de l'Observatoire de Grenoble, BP 53, 38041 Grenoble Cedex 9, France \\ 3 Institute de Radioastronomie Millimétrique, 300 rue de la Piscine, 38406 St Martin d'Hères Cedex, France \\ 4 INAF-Osservatorio Astrofisico di Arcetri, Largo E. Fermi 5, 50125 Firenze, Italy \\ ${ }^{5}$ Harvard-Smithsonian Center for Astrophysics, 60 Garden Street, Cambridge, MA 0213 \\ 6 Department of Physics and Astronomy, University of Victoria, Victoria, BC V8P 1A1, Canada \\ 7 National Research Council of Canada, Herzberg Institute of Astrophysics, 5071 West Saanich Road, Victoria, BC V9E 2E7, Canada \\ ${ }^{8}$ Leiden Observatory, PO Box 9513, 2300 RA Leiden, The Netherlands \\ 9 Max-Planck-Institut fur Radioastronomie, Auf dem Hügel 69, 53121 Bonn, Germany
}

Received 14 February 2007 / Accepted 29 March 2007

\begin{abstract}
Context. The transition between the low density groups of T Tauri stars and the high density clusters around massive stars occurs in the intermediate-mass $(\mathrm{IM})$ range $\left(M_{*} \sim 2-8 M_{\odot}\right)$. High spatial resolution studies of IM young stellar objects (YSO) can provide important clues to understand the clustering in massive star forming regions.

Aims. Our aim is to search for clustering in IM Class 0 protostars. The high spatial resolution and sensitivity provided by the new A configuration of the Plateau de Bure Interferometer (PdBI) allow us to study the clustering in these nearby objects.

Methods. We have imaged three IM Class 0 protostars (Serpens-FIRS 1, IC $1396 \mathrm{~N}$, CB 3) in the continuum at 3.3 and $1.3 \mathrm{~mm}$ using the PdBI. The sources have been selected with different luminosity to investigate the dependence of the clustering process on the luminosity of the source.

Results. Only one millimeter (mm) source is detected towards the low luminosity source Serpens-FIRS 1 . Towards CB 3 and IC1396 N, we detect two compact sources separated by $\sim 0.05 \mathrm{pc}$. The $1.3 \mathrm{~mm}$ image of IC $1396 \mathrm{~N}$, which provides the highest spatial resolution, reveal that one of these cores is splitted in, at least, three individual sources.
\end{abstract}

Key words. stars: formation - stars: individual: Serpens-FIRS 1 - stars: individual: IC 1396 N - stars: individual: CB 3

\section{Introduction}

Low and high mass stars $\left(M_{*}>8 M_{\odot}\right)$ are formed in different regimes. While low mass stars can be formed isolated or in loose associations, high mass stars are always found in tight clusters. Intermediate-mass young stellar objects (IMs) (protostars and Herbig Ae/Be [HAEBE] stars with $M_{*} \sim 2-8 M_{\odot}$ ) constitute the link between low- and high-mass stars. In particular the transition between the low density groups around T Tauri stars and the dense clusters around massive stars occurs in these objects. Testi et al. $(1998,1999)$ studied the clustering around HAEBE stars using optical and near-infrared (NIR) images and concluded that transition occurs smoothly from Ae to Be stars. Thus, these stars are key objects to study the onset of clustering.

Thus far, clustering has only been studied at infrared and optical wavelengths because of the limited spatial resolution and sensitivity of the $\mathrm{mm}$ telescopes. Thus, the earliest stages of the cluster formation were hidden to the observers. The subarcsecond angular resolution provided by the new A configuration of the PdBI allows, for the first time, to study clustering at $\mathrm{mm}$ wavelengths with a similar sensitivity and spatial resolution to the NIR studies. In this Letter, we present interferometric continuum observations of the IM protostars Serpens-FIRS 1 (precursor of a Ae star) and CB 3 (precursor of a Be star) aimed to study the clustering phenomena in the early Class 0 phase. We also use the data at highest spatial resolution towards IC $1396 \mathrm{~N}$ reported in this special issue by Neri et al. (Paper II, hereafter).

\subsection{Serpens-FIRS 1}

Serpens-FIRS 1 is a $46 L_{\odot}$ Class 0 source located in a very active star forming region. Previous mid-IR and NIR studies show that the population of YSOs is strongly clustered, with the Class I sources more clustered than the Class II ones (Kaas et al. 2004). The sub-clusters of Class I sources are located in a NW-SE oriented ridge following the distribution of dense cores in the molecular cloud with a subclustering spatial scale of $0.12 \mathrm{pc}$ (see Fig. 1). The Class II stars are located surrounding the molecular cores with a subclustering spatial scale of $0.25 \mathrm{pc}$. Adopting a distance of $310 \mathrm{pc}$, the YSOs density in the sub-clusters ranges from $360-780 \mathrm{pc}^{-2}$. Several high angular resolution $\mathrm{mm}$ studies have been made in the Serpens molecular cloud (Testi \& Sargent 1998; William \& Myers 1999; Hogerheijde et al. 1999; Testi et al. 2000). We have imaged at higher spatial resolution a region of $0.04 \mathrm{pc}$ around the intense mm-source FIRS 1 . 
Table 1. Millimeter flux densities, sizes, spectral indexes and masses.

\begin{tabular}{|c|c|c|c|c|c|c|c|c|c|c|}
\hline & \multicolumn{2}{|c|}{ Position } & \multirow[t]{2}{*}{$\begin{array}{c}\text { Peak } \\
\text { (mJy/beam) }\end{array}$} & \multirow[t]{2}{*}{$\begin{array}{c}\text { Gaussian width } \\
\text { (") }\end{array}$} & \multirow[t]{2}{*}{$\begin{array}{c}\text { Int. Intensity } \\
(\mathrm{mJy})\end{array}$} & \multirow[t]{2}{*}{$\begin{array}{r}\text { Mass }^{2} \\
\left(M_{\odot}\right) \\
\end{array}$} & \multirow[t]{2}{*}{$\begin{array}{l}\mathrm{Size}^{3} \\
(\mathrm{AU}) \\
\end{array}$} & \multirow[t]{2}{*}{$\alpha^{4}$} & \multirow[t]{2}{*}{$\begin{array}{c}\text { Sensitivity }^{5} \\
\left(M_{\odot}\right)\end{array}$} & \multirow[t]{2}{*}{$\begin{array}{c}\text { Sampled area }^{6} \\
r(\mathrm{pc})\end{array}$} \\
\hline Serpens & FIRS 1 & & & & & & & & & \\
\hline $1.3 \mathrm{~mm}$ & $18: 29: 49.80$ & $01: 15: 20.41$ & $273(1)$ & $0.50^{\prime \prime} \times 0.63^{\prime \prime}$ & 357 & 0.1 & 65 & 1.57 & 0.01 & 0.02 \\
\hline $3.3 \mathrm{~mm}$ & $18: 29: 49.80$ & $01: 15: 20.41$ & $63(0.5)$ & $0.80^{\prime \prime} \times 1.73^{\prime \prime}$ & 71 & & & & & 0.04 \\
\hline \multicolumn{11}{|c|}{ CB 3-1 } \\
\hline $1.3 \mathrm{~mm}$ & $00: 28: 42.60$ & $56: 42: 01.11$ & $20(1)$ & $0.36^{\prime \prime} \times 0.48^{\prime \prime}$ & 34 & 0.62 & 600 & 2.52 & 0.04 & 0.16 \\
\hline $3.3 \mathrm{~mm}$ & $00: 28: 42.60$ & $56: 42: 01.11$ & $2.0(0.5)$ & $0.88^{\prime \prime} \times 1.27^{\prime \prime}$ & 2.9 & & & & & 0.32 \\
\hline \multicolumn{11}{|l|}{ CB 3-2 } \\
\hline $1.3 \mathrm{~mm}$ & $00: 28: 42.20$ & $56: 42: 05.11$ & $10(1)$ & $0.31^{\prime \prime} \times 0.43^{\prime \prime}$ & 13 & 0.24 & 330 & 1.87 & 0.04 & 0.16 \\
\hline $3.3 \mathrm{~mm}$ & 00:28:42.20 & 56:42:05.11 & $2.1(0.5)$ & $0.80^{\prime \prime} \times 1.00^{\prime \prime}$ & 2.1 & & & & & 0.32 \\
\hline
\end{tabular}

${ }^{1}$ Half-power width of the fitted $2 \mathrm{D}$ elliptical Gaussian. ${ }^{2}$ Mass estimated using the $1.3 \mathrm{~mm}$ fluxes and assuming $T_{\mathrm{d}}=100 \mathrm{~K}$ and $\kappa_{1.3} \mathrm{~mm}=$ $0.01 \mathrm{~g}^{-1} \mathrm{~cm}^{2} .{ }^{3}$ Deconvolved source size at $1.3 \mathrm{~mm} .{ }^{4} 1.3 \mathrm{~mm} / 3.3 \mathrm{~mm}$ spectral index. ${ }^{5} 5 \times \mathrm{rms}$ mass sensitivity derived from the $1.3 \mathrm{~mm}$ image assuming $T_{\mathrm{d}}=100 \mathrm{~K}$ and $\kappa_{1.3 \mathrm{~mm}}=0.01 \mathrm{~g}^{-1} \mathrm{~cm}^{2} .{ }^{6}$ Radius $(H P B W / 2)$ of the PdBI primary beam at the source distance.

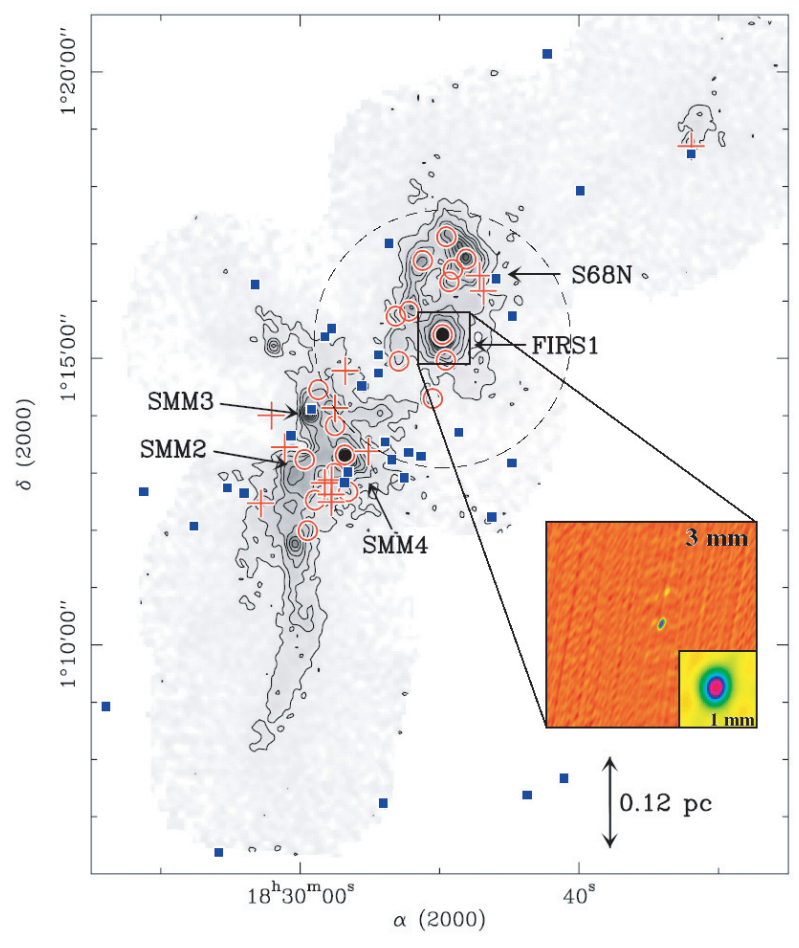

Fig. 1. Dust continuum mosaic (contours and grey scale) of the Serpens main core as observed with the IRAM $30 \mathrm{~m}$ telescope. The location of the Class II (blue filled squares), flat (red crosses) and Class I sources (red empty circles) is indicated (adapted from Kaas et al. 2004). In the inset, we show the $3 \mathrm{~mm}$ and $1.3 \mathrm{~mm}$ (small inset) continuum images observed with the PdBI. Note that only one compact core is detected in this region down to a spatial scale of less than $100 \mathrm{AU}$. The dashed circle marks a region of $0.2 \mathrm{pc}$ radius around FIRS 1 .

\subsection{IC $1396 \mathrm{~N}$}

IC $1396 \mathrm{~N}$ is a $\sim 300 L_{\odot}$ source located at a distance of $750 \mathrm{pc}$ (Codella et al. 2001). A total population of $\sim 30$ YSOs has been found in this region (Getman et al. 2007; Nisini et al. 2001). These YSOs present an elongated spatial distribution with an age gradient towards the center of the Class I/0 system. The Class III sources are located in the outer rim of the globule, the Class II sources are congregated in the bright ionized rim and the Class I/0 objects are located towards the dense molecular clump (see Fig. 2). The average density of YSOs in the globule is $\sim 200 \mathrm{pc}^{-2}$. We have mapped a region of $0.1 \mathrm{pc}$ around the Class 0/I system.

\section{3. $C B 3$}

CB 3 is a large globule $\left(930 L_{\odot}\right)$ located at $2.5 \mathrm{kpc}$ from the Sun (Codella \& Bachiller 1999). A strong submillimeter source is observed in the central core (see Fig. 3 and Huard et al. 2000). Deep NIR images of the region show 40 NIR sources, from which at least 22 are very red, indicative of pre-main sequence stars (Launhardt et al. 1998). Up to our knowledge, there are no mid-IR and/or X-ray studies in this region. Then, the census of YSOs is not complete in this IM source. We have mapped a region of $0.32 \mathrm{pc}$ around the submillimeter source.

\section{Observations}

The observations were made on January and February, 2006. The spectral correlator was adjusted to cover the entire RF passbands $(580 \mathrm{MHz})$ for highest continuum sensitivity. The overall flux scales for each epoch and for each frequency band were set on 3C 454.3 and MWC349 (for CB 3), and 1749+096 (for SerpensFIRS 1). The resulting continuum point source sensitivities ( $5 \times \mathrm{rms}$ ) were estimated to $2.00 \mathrm{mJy}$ at $237.571 \mathrm{GHz}$ and $0.5 \mathrm{mJy}$ at $90.250 \mathrm{GHz}$ for $\mathrm{CB} 3$ and $40.00 \mathrm{mJy}$ at $237.571 \mathrm{GHz}$ and $7.0 \mathrm{mJy}$ at $90.250 \mathrm{GHz}$ for Serpens-FIRS1. The corresponding synthesized beams adopting uniform weighting were $0.4^{\prime \prime} \times 0.3^{\prime \prime}$ at $237.571 \mathrm{GHz}$ and $1.0^{\prime \prime} \times 0.8^{\prime \prime}$ at $90.250 \mathrm{GHz}$ for $\mathrm{CB} 3$ and $0.6^{\prime \prime} \times 0.4^{\prime \prime}$ at $237.571 \mathrm{GHz}$ and $1.7^{\prime \prime} \times 0.7^{\prime \prime}$ at $90.250 \mathrm{GHz}$ for Serpens-FIRS 1. (See Paper II for IC 1396 N.)

\section{Results}

In Table 1 we present the coordinates, sizes and mm fluxes of the compact cores detected in Serpens-FIRS 1 and CB 3. The results towards IC $1396 \mathrm{~N}$ are presented in Paper II. Only $1 \mathrm{~mm}-$ source is detected in Serpens-FIRS 1 down to a separation of less than $100 \mathrm{AU}$. The other targets turned out to be multiple sources. We have detected $2 \mathrm{~mm}$-sources towards $\mathrm{CB} 3$ and $4 \mathrm{~mm}$-sources towards IC $1396 \mathrm{~N}$.

The 4 compact sources towards IC $1396 \mathrm{~N}$ are grouped in 2 sub-clusters separated by $0.05 \mathrm{pc}$ which are spatially coincident with the sources named BIMA 2 and BIMA 3 by Beltrán et al. (2002). The projected distance between these sub-clusters is similar to that found by Hunter et al. (2007) between the $\mathrm{mm}$ sub-clusters in the massive star forming region NGC 6336 I. This distance is also similar to the distance between the stars forming the Trapezium in Orion (from 5000 to $10000 \mathrm{AU}$ ). Thus it is a typical distance between the IM and massive stars in the 

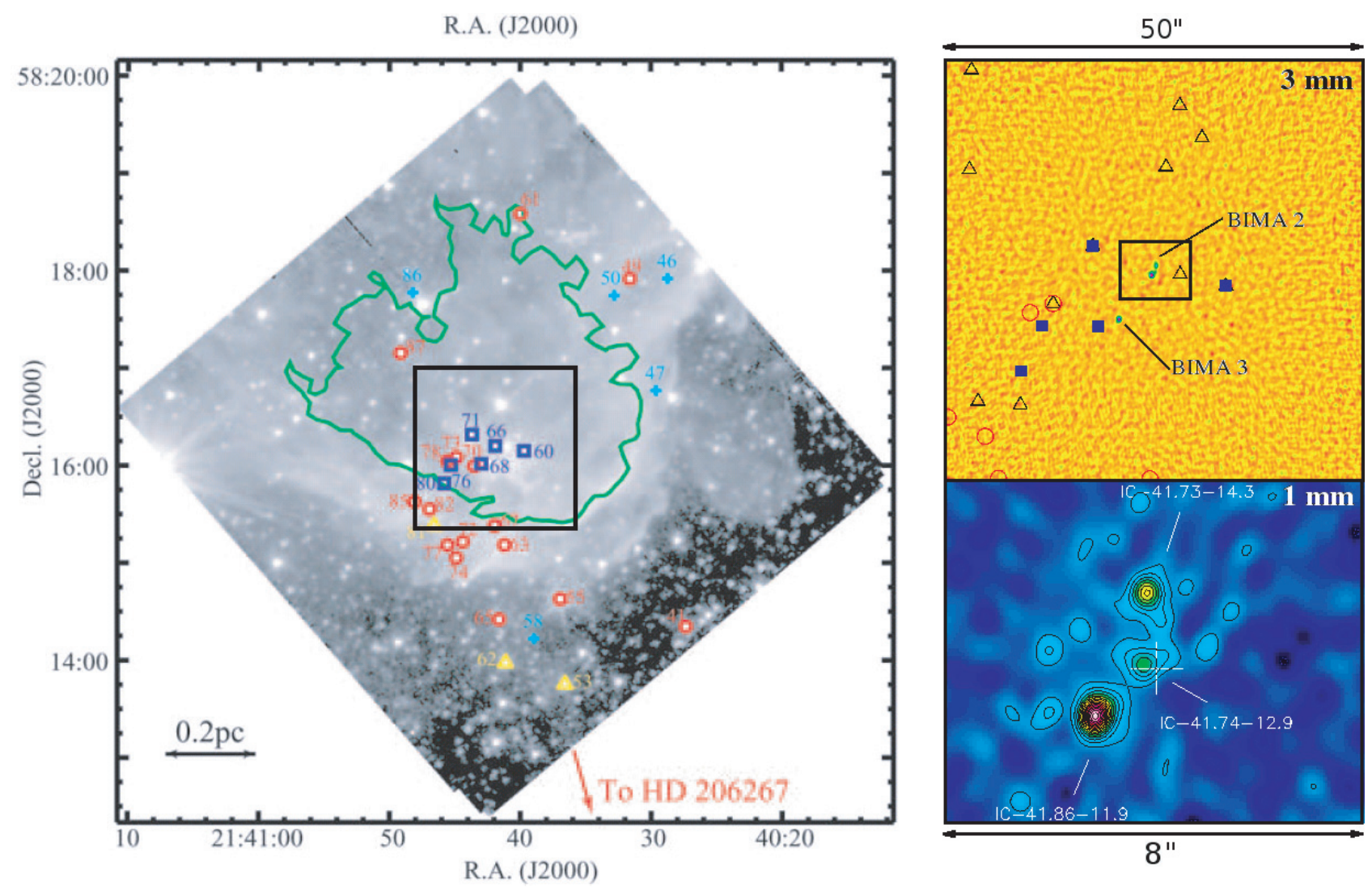

Fig. 2. On the left, we show the $5^{\prime} \times 5^{\prime}$ Spitzer IRAC $3.6 \mu \mathrm{m}$ image towards IC $1396 \mathrm{~N}$ (adapted from Getman et al. 2007). The location of the globule is marked by the green contour and the Class III (yellow triangles), Class II (red circles) and blue squares (Class 0/I) sources are indicated. On the right, we show the $3 \mathrm{~mm}$ (up) and $1.3 \mathrm{~mm}$ (down) continuum images observed with the PdBI. In the $3 \mathrm{~mm}$ image we also indicate the Class III (black triangles), Class II (red circles) and Class 0/I (filled blue squares) sources.

same cloud. Our high angular resolution observations reveal that BIMA 2 is itself composed of 3 compact cores embedded in a more extended component (see Fig. 2). These 3 compact cores are new $\mathrm{mm}$ detections and constitute the first sub-cluster of Class 0 IM sources detected thus far.

In CB 3 we have detected $2 \mathrm{~mm}$-sources separated by $0.06 \mathrm{pc}$ (see Table 1 and Fig. 3). These compact cores are new detections and the separation between them is similar to that between BIMA 2 and BIMA 3 in IC 1396 N. In fact, the structure of the globule CB 3 resembles much that of IC $1396 \mathrm{~N}$ but the angular resolution of our observations prevent us from resolving any possible sub-cluster of compact cores in this more distant source. Note that the masses of CB 3-1 and CB 3-2 are similar to that of the sub-cluster BIMA 2 (Paper II).

The number of detections is limited by the sensitivity of our observations. In Table 1 we show the point source mass sensitivity assuming a dust temperature of $100 \mathrm{~K}$ (typical for hot cores and circumstellar disks around luminous Be stars) and $\kappa_{1.3 \mathrm{~mm}}=0.01 \mathrm{~g}^{-1} \mathrm{~cm}^{2}$ for each target. It is possible that we miss a population of weak Class $0 / \mathrm{I}$ sources in $\mathrm{CB} 3$ where the mass sensitivity is poor $\left(0.04 M_{\odot}\right)$. However, the sensitivity in Serpens-FIRS $1\left(0.01 M_{\odot}\right)$ and IC1396 N $\left(0.007 M_{\odot}\right)$ is good enough to detect disks around early Be stars that usually have masses of $\sim 0.01 M_{\odot}$ (see e.g. Fuente et al. 2003, 2006). We should have also detected massive disks $\left(\sim 0.1 M_{\odot}\right)$ around Herbig Ae and T Tauri stars although the dust temperature is lower, $T_{\mathrm{d}}=15-56 \mathrm{~K}$ (Natta et al. 2000). But there is still the possibility of the existence of HAEBE or T Tauri stars with weak circumstellar disks that are not detected in our $\mathrm{mm}$ im- ages. Another possibility is that we are missing a population of hot corinos (we refer as "hot corino" to the warm material $(\sim 100 \mathrm{~K})$ around a low mass Class 0 protostar regardless of its chemical composition) with masses below the values reported in Table 1. Our sensitivity is good enough to detect a hot corino similar to IRAS $16293-2422 \mathrm{~A}$ and B $\left(L \sim 10 L_{\odot}\right)$ at the distance of our sources (see Bottinelli et al. 2004). Thus the possible "missed" hot corinos should correspond to lower luminosity protostars. Finally, we can be missing a population of dense and cold cores. Assuming a dust temperature of $10 \mathrm{~K}$, these compact cold cores should have masses of less than $0.17,0.12$ and $0.7 M_{\odot}$ in Serpens-FIRS 1, IC $1396 \mathrm{~N}$ and CB 3 respectively. These masses are not large enough to form new IM stars.

\section{Discussion}

Testi et al. (1999) studied the clustering around a large sample of HAEBE stars. In order to quantify the concept, they introduced the parameter $N_{k}$, defined as the number of stars in a radius of $0.2 \mathrm{pc}$, the typical cluster radius. They showed that rich clusters are only found around the most massive stars, although the parameter $N_{k}$ is highly variable. Some Be stars are born quite isolated, while others have $N_{k}>70$. For our sources this number is 22 (Launhardt et al. 1998, but the census is not complete), 29 (from Fig. 1) and 28 (Getman et al. 2007; Nisini et al. 2001) in CB3, Serpens and IC $1396 \mathrm{~N}$ respectively, where all previously known YSOs (Class 0, I, II and III) in the regions are considered. 


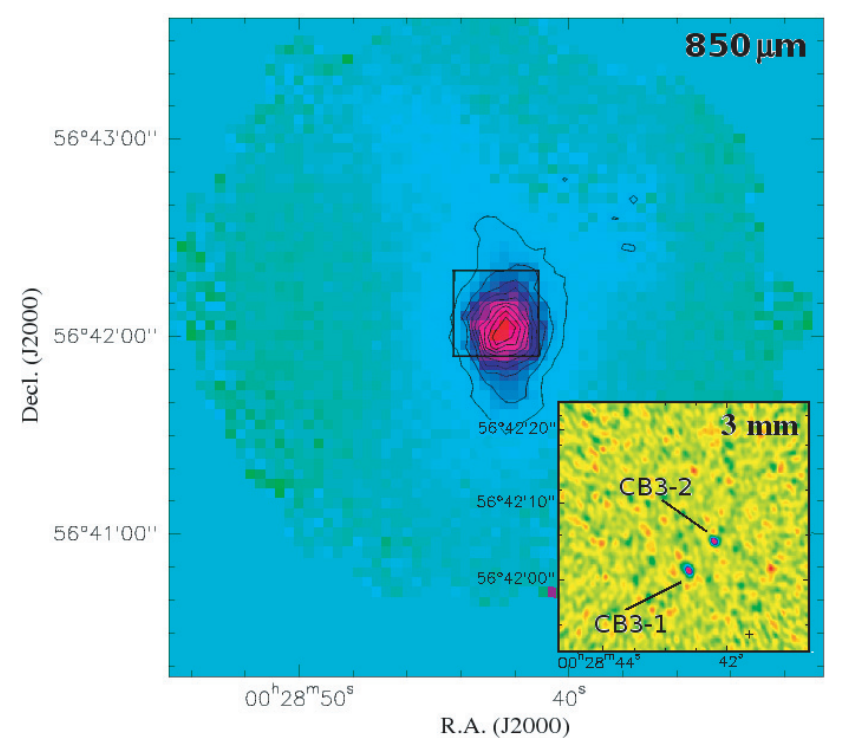

Fig. 3. Dust continuum emission at $850 \mu \mathrm{m}$ as observed with SCUBA towards CB 3. In the inset, we show the $3 \mathrm{~mm}$ continuum image observed with PdBI. Note that two compact cores are detected towards the single-dish peak.

Our maps show 2 sources in CB 3 on a 0.3 pc scale, 1 source in Serpens-FIRS 1 on a 0.04 pc scale, and 4 sources in IC $1396 \mathrm{~N}$ on a $0.1 \mathrm{pc}$ scale. Defining $N_{\mathrm{mm}}$ as the number of $\mathrm{mm}$ sources in a radius of $0.2 \mathrm{pc}$, we can estimate $N_{\mathrm{mm}}$ from our observations and provide a revised value for the total number of YSOs at this scale. In Serpens our interferometric observations do not add any new mm source to previous data. We have observed the most intense mm clump in Fig. 1, the most likely to be a multiple source, and only found 1 compact source. Based on the $30 \mathrm{~m}$ map shown in Fig. 1 and assuming that all the clumps host only one source we estimate $N_{\mathrm{mm}} \sim 7$ from a total of 29 YSOs. In CB 3, our data add 2 new mm sources $\left(N_{\mathrm{mm}}=2\right)$ to the previous census of YSOs based on NIR studies. In IC $1396 \mathrm{~N}$, we estimate $N_{\mathrm{mm}}=4-16$. The upper limit has been calculated assuming a constant density of $\mathrm{mm}$ sources in the region. Usually, the Class 0/I stars are not uniformly distributed in the clouds, but grouped in sub-clusters that are coincident with the peak of dense cores. Thus the value of $N_{\mathrm{mm}}$ is very likely close to 4 and we assumed this number hereafter. Since BIMA 2 and BIMA 3 were previously detected in the X-rays surveys by Getman et al. (2007), we only add two new sources (due to the multiplicity of BIMA 2) to the total number of YSOs in this region.

Summarizing, the total number of YSOs is now 29, 24 and 30 for Serpens-FIRS 1, CB 3 and IC 1396 N respectively. While Serpens-FIRS 1 is an extraordinarily rich cluster compared with the clusters around Ae stars reported by Testi et al. (1999), CB 3 and IC $1396 \mathrm{~N}$ do not seem to become one of the crowded clusters $\left(N_{k} \sim 70\right)$ detected by these authors around Be stars. However, this conclusion might not be true. The interferometer is only sensitive to dense and compact cores and provides a biased vision of the star forming regions. In fact our interferometric observations accounts for less than $1 \%$ of the total interstellar mass in the studied globules, i.e., $~ 10,58$ and $64 M_{\odot}$ are missed in Serpens-FIRS 1, CB 3 and IC 1396 N respectively (Alonso-Albi et al. 2007). One possibility is that this mass is in the form of many weak hot corinos which could eventually become low mass stars. The fate of these hot corinos is, however, linked to the evolution of the IM protostar that is progressively dispersing and warming the surrounding material (Fuente et al. 1998). Another possibility is that the "missed" mass is in the form of an extended and massive envelope. This envelope (if not totally dispersed by the IM star) could produce new stars in a forthcoming star formation event.

\section{Summary}

We have searched for clustering at mm wavelengths in 3 IM star forming regions. We have detected 1, 2 and 4 compact cores in Serpens-FIRS 1, CB 3 and IC $1396 \mathrm{~N}$ respectively. The compact cores are not distributed uniformly but grouped in sub-clusters separated by $\sim 0.05 \mathrm{pc}$. Such a separation is a typical distance for both IM and massive stars within the same cloud. We have used our mm observations to complete the census of YSOs in these regions and compare them with the clusters found by Testi et al. (1999) in the more evolved HAEBE stars. Serpens-FIRS 1 seems to belong to an extraordinarily rich cluster. The density of YSOs in the high luminosity sources IC $1396 \mathrm{~N}$ and CB 3 is consistent with the density found in the clusters around Be stars although our sources are not found between the most crowded regions. The large amount of interstellar gas and dust in the studied regions suggest that new star formation events are still possible.

Acknowledgements. We are grateful to Phil Myers for his careful reading of the manuscript. A.F. is grateful for support from the Spanish MEC and FEDER funds under grant ESP 2003-04957, and from SEPCT/MEC under grant AYA 200307584 .

\section{References}

Alonso-Albi, T., et al. 2007, in preparation

Beltrán, M. T., Girart, J. M., Estalella, R., Ho, P. T. P., \& Palau, A. 2002, ApJ, 573,246

Bottinelli, S., Ceccarelli, C., Neri, R., et al. 2004, ApJ, 617, L69

Codella, C., \& Bachiller, R. 1999, A\&A, 350, 659

Codella, C., Bachiller, R., Nisini, B., Saraceno, P., \& Testi, L. 2001, A\&A, 376, 271

Davis, C. J., Matthews, H. E., Ray, T. P., Dent, W. R. F., \& Richer, J. S. 1999, MNRAS, 309, 141

Fuente, A., Martín-Pintado, J., Bachiller, R., Neri, R., \& Palla, F. 1998, A\&A, 334,253

Fuente, A., Rodríguez-Franco, A., Testi, L., et al. 2003, ApJ, 598, L39

Fuente, A., Alonso-Albi, T., Bachiller, R., et al. 2006, ApJ, 649, L119

Getman, K. V., Feigelson, E. D., Garmire, G., Broos, P., \& Wang, J. 2007, ApJ, 654,316

Hogerheijde, M. R., van Dishoeck, E. F., Salverda, J. M., \& Blake, G. A. 1999, ApJ, 513, 350

Huard, T. L., Weintraub, D. A., \& Sandell, G. 2000, A\&A, 362, 635

Hunter, T. R., Brogan, C. L., Megeath, S. T., et al. 2006, ApJ, 649, 888

Kaas, A. A., Olofsson, G., Bontemps, S., et al. 2004, A\&A, 421, 623

Launhardt, R., Henning, T., \& Klein, R. 1998, in Star Formation with the Infrared Space Observatory, ed. J. L. Yun, \& R. Liseau (San Francisco: ASP), ASP Conf. Ser., 132, 119

Natta, A., Grinin, V., \& Mannings, V. 2000, Protostars and Planets IV, 559

Neri, R., Fuente, A., Ceccarelli, et al. 2007, A\&A, 468, L33

Nisini, B., et al. 2001, A\&A, 376, 553

Testi, L., \& Sargent, A. I. 1998, ApJ, 508, L91

Testi, L., Palla, F., \& Natta, A. 1998, A\&AS, 133, 81

Testi, L., Palla, F., \& Natta, A. 1999, A\&A 342, 515

Testi, L., Sargent, A. I., Olmi, L., \& Onello, J. S. 2000, ApJ, 540, L53

Williams, J. P., \& Myers, P. C. 1999, ApJ, 518, L37 